\title{
Fungos causadores de ferrugens (Uredinales) da Reserva Biológica do Lago Piratuba, Amapá, Brasil
}

Rust fungi (Uredinales) from Piratuba Lake Biological Reserve, Amapá state, Brazil

\author{
Isadora Fernandes de França ${ }^{1}$, Helen Maria Pontes Sotão ${ }^{2}$ \& Salustiano Vilar Costa-Neto ${ }^{3}$
}

\begin{abstract}
Resumo
Este trabalho apresenta resultados do levantamento da micota Urediniológica realizado na Reserva Biológica do Lago Piratuba e áreas de entorno, no estado do Amapá. Foram inventariados 17 táxons da ordem Uredinales, parasitando 15 gêneros de plantas hospedeiras, classificados em 12 famílias vegetais. As espécies Aecidium amazonense Henn, Phragmidiella bignoniacearum (W.T. Dale) Buriticá \& J.F. Hennen, Prospodium laevigatum J. F. Hennen \& Sotão, Puccinia cynanchi Berk. \& M. A. Curtis, Puccinia lateritia Berk. \& M. A. Curtis, Puccinia peperomiae J. C. Lindq., Puccinia puta H.S. Jacks. \& Holw. e Puccinia spegazzinii De Toni são novas citações para o Amapá. Todas as espécies inventariadas são primeiros registros para a área de estudo. Palavras-chave: Amazônia, Basidiomycota, Brasil, fungos, Urediniomycetes.
\end{abstract}

\begin{abstract}
This paper presents the results of the rust mycobiota (Uredinales) inventory from Lake Piratuba Biological Reserve, Amapá state, Brazil. Seventeen taxa of rust fungi were identified. The specimens were parasitizing fifteen host plant genera from twelve families. The species Aecidium amazonense Henn., Phragmidiella bignoniacearum (W.T Dale) Buriticá \& J.F. Hennen, Prospodium laevigatum J. F. Hennen \& Sotão, Puccinia cynanchi Berk. \& M. A. Curtis, Puccinia lateritia Berk. \& M. A. Curtis, Puccinia peperomiae J. C. Lindq., Puccinia puta H.S. Jacks. \& Holw. andPuccinia spegazzinii De Toni are new reports for Amapá. All the listed species are new reports for the study area.
\end{abstract}

Key words: Amazonia, Basidiomycota, Brazil, fungi, Urediniomycetes.

\section{Introdução}

Os fungos da ordem Uredinales (Urediniomycetes) são organismos parasitas obrigados, que apresentam uma alta especificidade em relação aos seus hospedeiros e que ocasionam uma doença vegetal comumente denominada como ferrugem.

Vastas áreas da América tropical, entre as quais está incluído o Brasil, permanecem até hoje sem estudos relativos a este grupo de doenças. Os trópicos brasileiros são regiões que apresentam ampla variação vegetacional, desde o semi-árido à florestas chuvosas e dos campos gerais ao cerrado, sendo, portanto, áreas potencialmente ricas em espécies de Uredinales, onde a realização de inventários pode adicionar informações essenciais para o aperfeiçoamento da sistemática desses fungos (Figueiredo \& Hennen 1998).

No estado do Amapá, podem ser ressaltados os trabalhos de Hennen \& Sotão $(1996,1997)$ e Hennen et al. (1998), nos quais foram publicadas novas espécies e variedades, e o de Sotão et al. (2001), que descreveram e ilustraram 15 espécies do gênero Puccinia. Para a área de estudo foram reportados dois novos registros de ferrugens sobre plantas em Fabaceae para o Brasil, Atelocauda incrustans Arthur \& Cummins e Chaconia alutacea Juel. (França \& Sotão 2009).

Este trabalho representa um inventário realizado sobre a micobiota da REBIO Lago Piratuba, estado do Amapá, tendo como objetivos contribuir para o

\footnotetext{
Universidade Federal Rural de Pernambuco, DEPA, Programa de Pós-Graduação em Fitopatologia, R. Dom Manoel de Medeiros s/n, Dois Irmãos, 52171-900, Recife, PE, Brasil. Autor para correspondência: isa_bio@yahoo.com.br.

${ }^{2}$ Museu Paraense Emílio Goeldi, MPEG, Coordenação de Botânica, C.P. 399, 66040-170, Belém, PA, Brasil.

${ }^{3}$ Centro de Pesquisa Científicas e Tecnológicas do Estado do Amapá, IEPA, Rod. Juscelino Kubitschek km 10, Fazendinha, 68912-250, Macapá, AP, Brasil.
} 
conhecimento da diversidade e ampliação da distribuição geográfica mundial das espécies de Uredinales, apresentando dados referentes a uma região com escasso conhecimento de sua biodiversidade.

\section{Material e Método}

Foram coletadas amostras com sintomas de ferrugem na Reserva Biológica (REBIO) do Lago Piratuba. Criada pelo Decreto No 84.914/1980 e alterado pelo Decreto $N^{\circ} 89.932 / 1984$, a reserva localiza-se no estado do Amapá entre as coordenadas geográficas $01^{\circ} 10^{\prime}-01^{\circ} 50^{\prime} \mathrm{N}$ e $49^{\circ} 34^{\prime}-0^{\circ} 34^{\prime} \mathrm{W}$, abrangendo uma área de aproximadamente 357.000 ha. Apresenta diversidade de formações vegetais e ecossistemas, abrigando algumas manchas de floresta tropical densa de planície aluvial, compreendendo a sub-região dos campos de planície do Amapá e a subregião do litoral (manguezal), bem como os ecossistemas de transição entre estas formações (IBAMA 2008).

As amostras foram coletadas em expedições realizadas em 2005 (novembro) e em 2006 (junho, julho e novembro), seguindo-se a metodologia de coleta, preservação e herborização de Cummins $\&$ Hiratsuka (2003).

A identificação dos espécimes foi baseada em literatura especializada, principalmente contendo a descrição dos táxons, tais como os trabalhos de Hennen et al. (2005), Buriticá(1999a,b), Carvalho Jr. et al. (2001) e Sotão et al . (2006). As ilustrações das espécies foram feitas em microscopia eletrônica de varredura (MEV) a partir de lâminas com estruturas dos fungos estudados.

Os espécimes estão depositados nos Herbários João Murça Pires (MG), do Museu Paraense Emílio Goeldi, Belém (PA) e Herbário Amapaense (HAMAB), do Instituto de Estudos e Pesquisas do Estado do Amapá, Macapá (AP).

Foram elaboradas chaves de identificação para as espécies estudadas que ocorrem na mesma família de planta hospedeira. Devido à especificidade de hospedeiro, própria deste grupo, os resultados estão apresentados primeiramente por família de hospedeiros, seguido das espécies de Uredinales relacionadas a cada família. São também informados os protólogos, espécimes estudados, distribuição geográfica do fungo e informações adicionais das espécies.

\section{Resultados e Discussão}

A partir dos inventários de fungos Uredinales realizados na Reserva e áreas de seu entorno, foram identificadas 17 espécies pertencentes a nove gêneros. Os espécimes registrados estavam parasitando 15 gêneros de plantas hospedeiras, distribuídos em 12 famílias vegetais.

Famílias Botânicas hospedeiras e respectivas ferrugens ocorrentes na REBIO do Lago Piratuba

\section{Annonaceae}

1.1 Aecidium amazonense Henn., Hedwigia 43: 169. 1904.

Fig. 1a-c

Material estudado: Sobre Guatteria sp. BRASIL. AMAPÁ: Tartarugalzinho, Base da Extrema, 14.XI.2005, H. Sotão \& L. Lobato H63 (I) (HAMAB, MG).

Ocorre no Brasil (Amazonas e Pará) e Peru (Hennen et al. 2005). Este é o primeiro registro desta espécie para o estado do Amapá.

Seis das onze espécies de Aecidium conhecidas sobre gêneros da família Annonaceae no neotrópico ocorrem sobre Guatteria spp. No entanto, Hennen et al. (2005) reconhecem apenas duas espécies, A. amazonense e A. guatteriae Dietel, pois consideram que as diferenças apresentadas entre estas seis espécies não são consistentes para diferenciá-las.

Aecidium amazonense pode ser identificada por apresentar células peridiais de parede externa lisa e interna verrucosa e eciósporos catenulados com parede de $2 \mu \mathrm{m}$ de espessura, verrucosa, contendo 3-4 grânulos refrativos grandes e um ou dois menores. Em A. guatteriae as células peridiais possuem parede externa estriada e interna verrucosa, e os esporos apresentam numerosos grânulos refrativos.

\section{Asclepiadaceae}

2.1 Puccinia cynanchi Berk. \& M.A. Curtis, J. Philadelphia Acad. Sci., ser. 2, 2: 281. 1853.

Fig. 1d

Material estudado: Sobre Ditassa sp., BRASIL. AMAPÁ: Amapá, Fazenda Macarry, 12.XI.2006, L.C. Lobato \& P. Sanjuan LC31 (III) (HAMAB, MG)

Esta espécie é referida para a Argentina, Cuba, Estados Unidos, Paraguai e Suriname (Lindquist 1982). No Brasil já foi relatada nos estados de Mato Grosso, Minas Gerais, Paraná, Pará, Rio de Janeiro, Rio Grande do Sul, Santa Catarina e São Paulo (Hennen et al. 2005). Esta é a primeira citação da espécie para o Estado do Amapá.

Tem sido reportada com diferentes sinonímias parasitanto, pelo menos, sete gêneros de Asclepiadaceae. No Brasil, Hennen et al. (2005) 

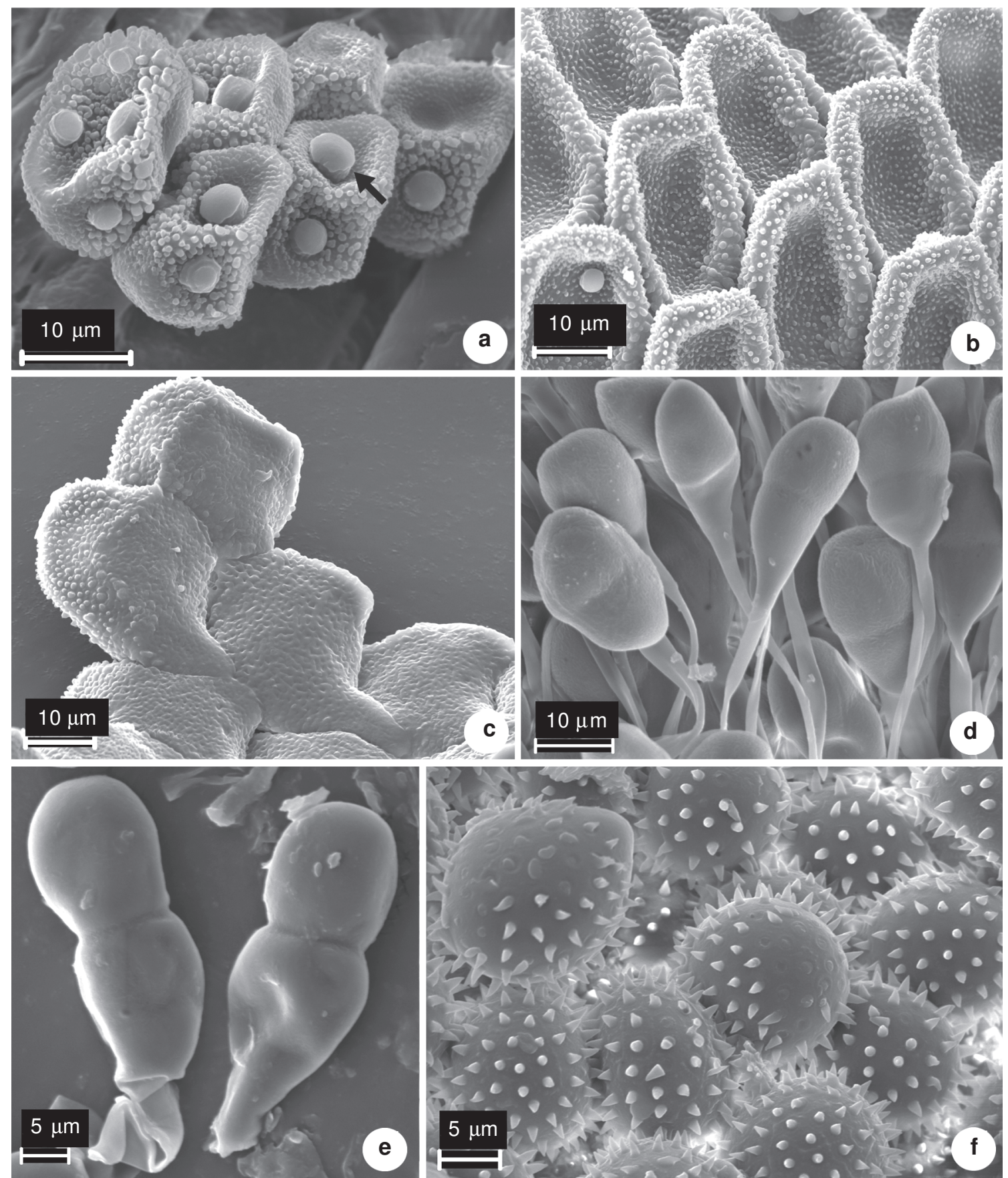

Figura 1 - a-c. Aecidium amazonense Henn - a. eciósporos de parede verrucosa com grânulos refrativos (seta); b-c. células peridiais, superfície interna verrucosa e externa lisa, respectivamente. d. Puccinia cynanchi Berk. \& M.A. Curtis - teliósporos, variação na forma dos esporos e na inserção do pedicelo. e. P. spegazzinii De Toni - teliósporos. f. Phragmidiella bignoniacearum (Dale) Buriticá \& J.F. Hennen - urediniósporos.

Figure 1 - a-c. Aecidium amazonense Henn - a. aeciospores wall verrucose with refractive granules (arrow); b-c. peridial cells, inner surface verrucose and outer surface smooth, respectively. d. Puccinia cynanchi Berk. \& M.A. Curtis - teliospores, variation in the spores shape and pedicel attachment. e. P. spegazzinii De Toni - teliospores. f. Phragmidiella bignoniacearum (Dale) Buriticá \& J.F. Hennen - urediniospores. 
citam a sua ocorrência sobre os gêneros Calotropis, Ditassa, Metastelma e Oxypetalum.

Sobre Asclepiadaceae são conhecidas as espécies Puccinia cynanchi (P. obliqua), P. rouliniae Henn. e $P$. araujae Lév., as quais são de difícil identificação devido às características morfológicas muito semelhantes. Jørstad (1956) propôs uma chave de identificação para estas espécies, onde P. cynanchi diferencia-se das outras por possuir teliósporos de largamente elipsóides a quase globosos, paredes usualmente de coloração marrom-canela, pedicelo geralmente inserido obliquamente e teliossoros marrom-escuros.
Segundo Lindquist (1982), a presença de teliósporos globosos contendo septos dispostos obliquamente é a principal característica de $P$. cynanchi, porém este é um aspecto variável e, em alguns casos, torna-se difícil diferenciá-la de $P$. araujae e $P$. roulinia. Através das imagens de microscopia eletrônica de varredura (MEV), observou-se que a parede dos teliósporos não é totalmente lisa, apresentando finas estrias. As imagens em MEV de teliósporos dos espécimes tipos destas três espécies citadas serviriam para comparar a ornamentação da parede com esta característica observada no espécime estudado.

\section{Asteraceae}

\section{Chave de identificação das espécies de Uredinales sobre plantas da família Asteraceae}

1. Sobre Mikania sp.; teliósporos cilíndricos a clavados, paredes com 0,5-1 $\mu \mathrm{m}$ de espessura lateral e 2-3 (-4) $\mu \mathrm{m}$ de espessura no ápice. 3.2. Puccinia spegazzinii

1'. Sobre Spilanthes sp.; teliósporos oblongos, paredes com 1-2,5 $\mu \mathrm{m}$ de espessura lateral e 4-16 $\mu \mathrm{m}$ de espessura no ápice

3.1. Puccinia cnici-oleracei

3.1 Puccinia cnici-oleracei Pers. ex Desm., Catal. Pl. Omis. p. 24. 1823.

Material estudado: Sobre Spilanthes sp., BRASIL. AMAPÁ: Amapá, REBIO do Lago Piratuba, Tabaco, 7.XI.2005, H. Sotão \& L.C. Lobato H03 (III) (HAMAB, MG); 30.VI.2006, I. França II7 (III) (HAMAB, MG).

Ocorre desde o Alasca e sul do Canadá até a Costa Rica, América do Sul, Europa e Ásia (Cummins 1978). No Brasil está distribuída nos estados do Acre, Amapá, Amazonas, Bahia, Ceará, Maranhão, Mato Grosso, Mato Grosso do Sul, Minas Gerais, Pará, Paraíba, Rio de Janeiro, Rio Grande do Sul e São Paulo (Hennen et al. 2005).

Puccinia cnici-oleracei é uma ferrugem microcíclica, com vários sinônimos encontrados na literatura. O patógeno tem sido registrado sobre muitas espécies de Asteraceae, família com grande número de membros, complexa e com ampla distribuição geográfica. Hennen et al. (2005) citam sete gêneros de Asteraceae parasitados por $P$. cnici-oleraceae para o Brasil: Acanthospermum, Eleutheranthera, Emilia, Melampodium, Picrosia, Spilanthes e Stenachaenium.

Puccinia cnici-oleracei foi relatada pela primeira vez para o Amapá por Sotão et al. (2001), parasitando plantas dos gêneros Eleutheranthera, Emilia, Melampodium e Spilanthes. Estes autores ressaltam que, na Amazônia, esta ferrugem é considerada de interesse econômico por parasitar o “jambu” (Acmella oleracea (L.) R. K. Jansen), cujas folhas são muito utilizadas na culinária regional.

3.2 Puccinia spegazzinii De Toni, in Saccardo, Syll. Fung. 7: 704. 1888. Fig. 1e Material estudado: Sobre Mikania sp., BRASIL. AMAPÁ: Tartarugalzinho, Base da extrema, 14.XI.2005, H. Sotão \& L. Lobato H68 (III) (HAMAB, MG).

Segundo Cummins (1978) é reportada desde o sul dos Estados Unidos até o Panamá e América do Sul. No Brasil é encontrada no Amazonas, Distrito Federal, Minas Gerais, Pará, Paraná, Rio de Janeiro, Santa Catarina, São Paulo e Rio Grande do Sul (Hennen et al. 2005). Este é o primeiro registro desta espécie para o estado do Amapá.

Segundo Viégas (1945) esta é uma ferrugem microcíclica, e os seus teliósporos germinam sem período de dormência. Evans \& Ellison (2005) confirmaram o ciclo de vida reduzido desta espécie através de inoculações experimentais.

Várias espécies de Mikania Willd. são parasitadas por P. spegazzinii. No Brasil, esta espécie tem sido reportada sobre $M$. cordifolia (L.f.) Willd., M. micrantha Kunth, M. scandens (L.) Willd. e $M$. smilacina DC.

Quatro espécies válidas de Puccinia são conhecidas sobre Mikania: P. granchacoensis Jørst., 
P. mikaniae H.S. Jacks. \& Holw., P. mikanifolia H.S. Jacks. \& Holw. e P. spegazzinii. Estaúltima diferencia-se por apresentar soros compactos, firmemente agrupados, coloração marrom acinzentada, tornandose branco acinzentado devido à germinação sem dormência dos teliósporos. Barreto \& Evans (1995) citam outras diferenças morfológicas entre estas espécies de Puccinia em relação a $P$. spegazzinii: $P$. granchacoensis apresenta teliósporos de coloração diferente (marrom-castanho claro) e possui um estágio uredinial conhecido e P. mikaniae tem o télio rodeado por écios.
Entre as espécies de Uredinales conhecidas sobre o gênero Mikania, Aecidium mikaniae Henn., Chrysocyclus mikaniae (Arthur) Syd., P. mikaniae, P. mikanifolia, P. spegazzini e Uromyces mikaniae Viégas estão registradas para o Brasil (Hennen et al. 2005). Em A. mikaniae não é produzida a fase teleomorfica. A espécie $C$. mikaniae diferencia-se de $P$. spegazzinii por apresentar télios agrupados em círculos, laranjaavermelhado, que se tornam cinza após a germinação; U. mikaniae diferencia-se por possuir teliósporos unicelulares e estágio ecial conhecido.

\section{Bignoniaceae}

\section{Chave de identificação das espécies de Uredinales sobre plantas da família Bignoniaceae}

1. Soros anamórficos produzindo esporos de parede bilaminada; télios com teliósporos bicelulares fortemente constritos no septo e isolados 4.2. Prospodium laevigatum

1'. Soros anamórficos produzindo esporos de parede simples; télios desconhecidos ou quando produzidos com teliósporos unicelulares e catenulados

2. Sobre Cydista sp.; uredínios com paráfises periféricas, urediniósporos 17,5-25×15-20 mm e com poros germinativos obscuros; teliósporos catenulados .......4.1. Phragmidiella bignoniacearum

2'. Sobre Memora sp.; uredínios sem paráfises, urediniósporos 26-31(-33)× 17-23(-24) mm e com 2 poros germinativos mais ou menos equatoriais; teliósporos desconhecidos

4.1 Phragmidiella bignoniacearum (W.T. Dale) Buriticá \& J.F. Hennen, Rev. Acad. Colomb. Cienc. 23: 425. 1999.

Fig. 1f

Anamorfo: Macabuna daleae Buriticá \& J.F. Hennen, Rev. Acad. Colomb. Cienc. 23: 425. 1999.

Material estudado: Sobre Cydista sp. (Bignoniaceae), BRASIL. AMAPÁ: Amapá, REBIO do Lago Piratuba, Tabaco, 07.XI.2005, H. Sotão \& L.C. Lobato H06 (II) (HAMAB, MG); Rego do Boiado, 11.VI.2006, CostaNeto et al. 2418 (II) (HAMAB, MG).

Ocorre em Trinidad, Panamá (Buriticá 1999b), no Brasil no estado do Pará (Sotão et. al. 2006). Esta é a primeira referência desta espécie para o Amapá.

Buriticá (1999b) transferiu o gênero desta espécie de Cerotelium para Phragmidiella e nomeou o seu anamorfo Macabuna daleae.

De acordo com Buriticá (1999b), $P$. bignoniacearum tem como hospedeiros os gêneros Clytostoma e Cydista. Para o Brasil, Hennen et al. (2005) citam Prospodium singeri Petr. parasitando o gênero Clytostoma.

Phragmidiella bignoniacearum pode ser diferenciada por apresentar teliósporos unicelulares e catenulados, enquanto em $P$. singeri os esporos são bicelulares e pedicelados. Esta espécie foi referenciada pela primeira vez para o Brasil, no estado do Pará, por Sotão et al. (2006), constituindo o primeiro registro de ferrugem sobre o gênero Cydista.

4.2 Prospodium laevigatum J. F. Hennen \& Sotão, Sida 17: 182. 1996.

Fig. 2a

Material estudado: Sobre Bignoniaceae indeterminada, BRASIL. AMAPÁ: Amapá, REBIO do Lago Piratuba, Piranhas, atrás da base do Ibama, 11.XI.2005, H. Sotão \& L.C. Lobato H48 (II, III) (HAMAB, MG).

Esta espécie tem distribuição conhecida apenas para o Brasil, estado do Pará (Hennen \& Sotão 1996; Sotão et al. 2009), sendo este o primeiro registro desta espécie para o estado do Amapá.

4.3 Uredo amapaensis J.F. Hennen \& Sotão, SIDA 17(1): 179-180. 1996.

Material estudado: Sobre Memora sp., BRASIL. AMAPÁ: Amapá, REBIO do Lago Piratuba, Tabaco, 7.XI.2005, $H$. Sotão \& L.C. Lobato H05 (II) (HAMAB, MG).

Espécie conhecida somente para o norte do Brasil, nos estados do Amapá (Hennen \& Sotão 1996) e Pará (Sotão et al. 2009). 
Hennen \& Sotão (1996) sugerem que Uredo amapaensis está relacionada a quatro espécies de Porotenus sobre Memora sp. (P. bibasiporulus J.F. Hennen \& Sotão, P. biporus J.F. Hennen \& Sotão, $P$. concavus Viégas e P. memorae F.C. Albuq.). Entretanto diferencia-se por apresentar parede dos urediniósporos fortemente equinulada, com ornamentação mais ou menos uniforme e sem áreas lisas, as quais estão presentes nos urediniósporos das espécies de Porotenus.

Hennen et al. (2005) reportam cinco espécies de Prospodium com teliósporos de parede lisa: $P$. aequinoctialis (Holw.) Cummins sobre o gênero Cydista; P. cumminsii F. Kern \& Thurst. sobre Amphilophium; P. gentryi J.F. Hennen \& Cummins sobre Parmentiera, P. laevigatum J.F. Hennen \& Sotão sobre Mansoa, e $P$. venezuelanum F. Kern sobre uma provável espécie de Tabebuia. Destas, $P$. laevigatum é a única registrada para o Brasil, e pode ser diferenciada por apresentar eciósporos bilaminados e bicapitados, télios ciatiformes, supraestomatais, teliósporos fortemente constritos no septo, sendo as células quase globóides e pedicelos frágeis.

Embora a espécie tipo tenha sido identificada sobre o gênero Mansoa, Sotão et al. (2009) relatou a ocorrência desta espécie em três outros gêneros de Bignoniaceae: Memora, Pachyptera e Stizophyllum, coletados na Floresta Nacional de Caxiuanã, Pará. No Brasil, somente P. laevigatum é conhecida sobre os gêneros Mansoa e Pachyptera. Sobre o gênero Stizophyllum também é registrada a espécie Prospodium stizophylli H.S. Jacks. \& Holw., que se diferencia de $P$. laevigatum por apresentar parede dos teliósporos finamente rugosas, pedicelo com um septo e presença de numerosos mesosporos.

Sobre Memora sp. são conhecidas seis espécies de Uredinales: Porotenus bibasiporulus J.F. Hennen \& Sotão, Porotenus biporus J.F. Hennen \& Sotão, Porotenus concavus Viégas, Porotenus memorae F. C. Albuq., Prospodium anomalum H.S. Jacks. \& Holw., e Uredo amapaensis J.F. Hennen \& Sotão. Prospodium laevigatum pode ser diferenciado das espécies de Porotenus por seus eciósporos coronados, e de $P$. anomalum por seus teliósporos de parede lisa.

\section{Boraginaceae}

\subsection{Aecidium sp.}

Material estudado: Sobre Cordia sp., BRASIL. AMAPÁ: Tartarugalzinho, Base da Extrema, 14.XI.2005, H. Sotão \& L.C. Lobato H62 e H66 (0, I) (HAMAB, MG).

As características observadas nos espécimes estudados foram: espermogônios adaxiais, agrupados, dispostos em manchas arredondadas e escurecidas. Écios abaxiais, opostos aos espermogônios, agrupados em manchas escurecidas, dispersas e geralmente arredondadas, cupulados, esbranquecidos; células peridiais trapezoidais ou hexagonais, 22-30 $\times 17-23 \mathrm{~mm}$, parede externa lisa e parede interna verrucosa; eciósporos catenulados, elipsóides a poliédricos, 26-8 ×20-26 mm, parede 1,5-2 mm de espessura lateral, com até 5(6) $\mathrm{cm}$ de espessura no ápice, finamente verrucosa, hialina.

Hennen et al. (2005) citam quatro espécies de Uredinales sobre Cordia sp. para o Brasil: Aecidium brasiliense Dietel, Aecidium cordiae Henn., Aecidium lindavianum P. Syd. \& Syd. e Puccinia cordiae Arthur. Aecidium sp. diferencia-se de A. brasiliense e A. lindavianum por apresentar esporos espessos no ápice. Os eciósporos de Aecidium sp. apresentam dimensões semelhantes às dos esporos de $A$. cordiae, porém, as células peridiais A. cordiae tem parede externa lisa e interna verrucosatubercular. Embora os esporos sejam catenulados o anamorfo de $P$. cordiae não apresenta perídio, diferindo, portanto, do táxon estudado.

Dale (1955) descreveu a espécie Aecidium trinitense W.T. Dale sobre Cordia bicolor A. DC. para Trinidad e Tobago. Esta espécie apresenta células peridiais com parede externa lisa e interna verrucosatubercular, porém seus esporos (18-26×15-20 mm) são menores que os da espécie estudada. Novas coletas são necessárias para realização de estudos adicionais.

\section{Convolvulaceae}

\section{Chave de identificação das espécies de Uredinales sobre plantas da família Convolvulaceae}

1. Uredínios sem perídio, urediniósporos com parede uniforme (1-1,5 $\mu \mathrm{m})$; teliósporos unicelulares e sésseis 6.1. Coleosporium ipomoeae

1'. Uredínios com perídio, urediniósporos com parede de 1,5-2 $\mu \mathrm{m}$ de espessura lateral e engrossada no ápice (até $8 \mu \mathrm{m}$ de espessura); teliósporos bicelulares e pedicelados 6.2. Puccinia puta 
6.1 Coleosporium ipomoeae (Schwein.) Burrill, Bull. Illinois State Lab. Nat. Hist. 2:217. 1885.

Sinanamorfos: Caeoma ipomoea Link em Willdenow, Sp. Pl. 6(2): 14. 1822. Peridermium ipomoeae Hedgec., Mycologia 9: 239. 1917.

Material estudado: Sobre Ipomoeae alba L. (Convolvulaceae), BRASIL. AMAPÁ: Amapá, REBIO do Lago Piratuba, Escavado do Jaburu, 8.VI.2006, S. Costa-Neto et al. 2387, 2388 (II) (HAMAB, MG).

Ocorre nas Américas da Argentina até os Estad os Unidos. No Brasil nos estados do Amapá, Minas Gerais, Pará, Piauí, Rio de Janeiro, São Paulo (Hennen et al.2005) e Mato Grosso (Vieira et al. 2004).

Para o Brasil, Hennen et al. (2005) citam cinco espécies de Uredinales sobre Ipomoea (Convolvulaceae): Coleosporium ipomoeae, Puccinia crassipes Berk. \& M. A. Curtis, Puccinia opulenta Spegazzini, Puccinia puta H.S. Jacks. \& Holw. ex F. Kern, Thurst. \& Whetzel e Uromyces vicinus H.S. Jackson e Holway. C. ipomoeae diferencia-se das espécies do gênero Puccinia e Uromyces, por apresentar teliósporos sésseis.

6.2 Puccinia puta H.S. Jacks. \& Holw. ex F. Kern, Thurst. \& Whetzel, Mycologia 25: 477. 1933.

Fig. 2 b-d

Anamorfo: Aecidium distinguendum P. Syd. \& Syd., Monogr. Ured. 4: 131. 1923.

Material estudado: Sobre Ipomoea fistulosa Mart. ex Choisy, BRASIL. AMAPÁ: Cutias, 05.XI.2005, H. Sotão \& L. Lobato H01 (II) (HAMAB, MG); sobre Ipomoea sp., Fazenda Macarry, 11.XI.2006, L. Lobato \& P. Sanjuan LC25 (II, III) (HAMAB, MG).

Esta espécie está registrada para o Brasil (Amazonas, Bahia e Ceará), Colômbia, Equador, México, Peru, Porto Rico e Venezuela (Hennen et al. 2005; Hernández 2006; Hennen \& McCain 1993). Este é o primeiro registro desta espécie para o estado do Amapá.

Nos comentários da espécie Coleosporium ipomoeae estão descritas as diferenças entre as espécies registradas sobre Ipomoea para o Brasil.

Para o Neotrópico, são conhecidas dez espécies de Puccinia que ocorrem sobre Ipomoea: $P$. crassipes Berk. \& M.A. Curtis, P. ibrae J.F. Hennen \& J.W. McCain, $P$. insignis Holw., P. jalapensis Cummins, P. megalospora (Orton) Arthur \& J.R. Johnst., P. nocticolor Holw., P. opulenta Speg., P. puta, P. rubicunda Holw. e P. sup erflua Holw. P. puta pode ser diferenciada de $P$. jalapensis por apresentar uredínio catenulado verrucoso, enquanto a segunda possui uredínio pedicelado equinulado. Puccinia crassipes, P.insignis, P.megalospora, $P$. opulenta,
$P$. rubicunda e $P$. superflua apresentam urediniósporos com parede uniforme, enquanto em $P$. puta estes esporos são mais espessados no ápice (até $8 \mathrm{~mm}$ ). Puccinia nocticolor possui urediniósporos de parede tuberculada, sendo verrucosa em $P$. puta. $P$. ibrae apresenta a parede dos teliósporos com 6-9 mm de espessura no ápice, e $P$. puta possui parede com 5-6 mm de espessura no ápice.

\section{Dioscoreaceae}

7.1 Sphenospora pallida (G. Winter) Dietel, Engler e Prantl., Nat.11:70. 1897.

Anamorfo: Uredo dioscoreae Henn., Hedwigia 35: 255.1896.

Material estudado: Sobre Dioscorea sp., BRASIL. AMAPÁ: Amapá, REBIO do Lago Piratuba, Rio Araguari, 5.VII.2006, I. França I31 (II) (HAMAB, MG).

Ocorre em Belize, Brasil (Acre, Amapá, Mato Grosso, Minas Gerais, Pará, Paraná, Rio de Janeiro, Santa Catarina e São Paulo), Equador e Venezuela (Hennen et al. 2005).

Albuquerque (1971) descreveu Uredo aristolochiae F.C. Albuq., e, posteriormente, Hennen et al. (1982) colocaram este táxon como sinônimo de $S$. pallida. Hennen et al. (2005) relatam a existência de um homônimo anterior denominado Uredo aristolochiae DC., sinônimo de Puccinia aristolochia (DC.) G. Winter, de ocorrência na Europa, diferente da espécie descrita por Albuquerque (1971). No entanto, é necessário um minucioso estudo da espécie de Albuquerque para confirmação do hospedeiro e do fungo.

Hennen et al. (2005) citaram duas espécies de Uredinales sobre o gênero Dioscorea no Brasil, Sphenospora pallida e Uredo dioscoreicola F. Kern, Cif. \& Thurst., as quais apresentam poucas diferenças entre seus urediniósporos, sendo a parede destes densamente equinulada em S. pallida e moderadamente equinulada em $U$. dioscoreicola. A melhor característica para identificar a espécie Sphenosporra pallida são os teliósporos bicelulares e septo vertical.

\section{Euphorbiaceae}

8.1 Phakopsora arthuriana Buriticá \& J.F. Hennen em: Buriticá, Rev. I.C.N.E. (Medellin) 5(2): 180. 1994. Anamorfo: Malupa jatrophicola (Arthur) Buriticá \& J.F. Hennen.

Material estudado: Sobre Jatropha sp., BRASIL. AMAPÁ: Amapá, REBIO do Lago Piratuba, Margem do Rio Araguari, 10.XI.2005, H. Sotão \& L.C. Lobato H31 (II) (HAMAB, MG). 
Segundo Buriticá (1999a) distribui-se pelas Antilhas, Brasil (São Paulo e Pará), Costa Rica, Cuba, El Salvador, Estados Unidos, Guatemala, Haiti, México, Panamá, Porto Rico, República Dominicana e Venezuela. Este é o primeiro registro desta espécie para o estado do Amapá.

Na revisão do gênero Phakopsora no neotrópico realizada por Buriticá (1999a), são citadas dez espécies parasitando quatro gêneros da família Euphorbiaceae. Porém, somente Phakopsora arthuriana e seu anamorfo estão citados ocorrendo sobre o gênero Jatropha.

Hennen et al. (2005) citam para o Brasil três espécies de Uredinales sobre o gênero Jatropha: Phakopsora arthuriana, Uromyces cnidoscoli Henn. e Uromyces jatrophicola Henn. Phakopsora arthuriana apresenta télio subepidermal, não irrompentes com 6 a 12 camadas de esporos sésseis enquanto as espécies de Uromyces apresentam telios irrompentes, teliósporos pedicelados e não em camadas.

\section{Piperaceae}

10.1 Puccinia peperomiae J.C. Lindq., Rev. Facult. Agr. La Plata. 29: 41. 1952.

Fig. 2e

Anamorfo: Uredo peperomiae Henn., Hedwigia Beiblatt 38: (69). 1899.

Material estudado: Sobre Piper sp., BRASIL. AMAPÁ: Tartarugalzinho, Base da Extrema, 14.XI.2005, H. Sotão \& L. Lobato H65 (III) (HAMAB, MG).

Ocorre na Argentina, Peru, Colômbia, Porto Rico e Brasil (Pará, Rio de Janeiro e São Paulo) (Hennen et al. 2005). Esta é a primeira citação desta espécie para o estado do Amapá.

Berndt et al. (2002) publicaram Crossopsora piperis R. Berndt, F.O. Freire \& C.N. Bastos sobre Piper hostmannianum (Miq.) C. DC., a qual tem como anamorfo Malupa piperinum (Syd.) Buriticá e J.F. Hennen. C. piperis diferencia-se de $P$. peperomiae por apresentar teliósporos arranjados em fileiras delgadas e pela presença de paráfises periféricas tanto nos uredínios como nos télios. Este representa o primeiro registro de télios para o Brasil.

\section{Poaceae}

\section{Chave de identificação das espécies de Uredinales sobre plantas da família Poaceae}

1. Sobre Guadua sp.; teliósporos unicelulares, catenulados; urediniósporos (21-)23-28(-31) × (14-) 16$19(-21) \mu \mathrm{m}, 4-5$ poros germinativos 11.1. Kweilingia divina

1'. Sobre Pariana sp.; teliósporos bicelulares, pedicelados; urediniósporos (15-)17-27 ×(13-)15-20 $\mu \mathrm{m}$, poros germinativos obscuros 11.2. Puccinia bambusarum

11.1 Kweilingia divina (Syd.) Buriticá, Rev. Acad. Colombiana Cienc. 22: 330. 1998. (0, I, II, III).

Sinanamorfos: Physopella inflexa (S. Ito) Buriticá \& J. F. Hennen, Rev. Acad. Colombiana Cienc. 19: 56. 1994. Aecidium thaungii A.A. Carvalho, J.F. Hennen \& Figueiredo, Summa Phytopatol. 27:261. 2001

Material estudado: Sobre Guadua sp., BRASIL. AMAPÁ: Amapá, REBIO do Lago Piratuba, Fazenda Monte Sião, 1.VII.2006, I. França I23 (II) (HAMAB, MG).

Esta espécie é reportada para a Índia, Japão e Taiwan (Cummins 1971). No Brasil, tem sido registrada para os estados do Amapá, Mato Grosso do Sul, Pará, Pernambuco, Rio de Janeiro e São Paulo (Hennen et al. 2005).

Carvalho Jr. et al. (2001) estudaram a fase teleomórfica da ferrugem encontrada sobre Bambusa vulgaris Schrad. exJ.C. Wendl., coletadas em São Paulo, Brasil, e propondo Kweilingia divina (Syd.) Buriticá, como denominação correta para esta espécie. Estes autores também validaram dois anamorfos, Physopella inflexa (S. Ito) Buriticá e J.F. Hennen, como fase uredinial e Aecidium thaungii A.A. Carvalho, J.F. Hennen \& Figueiredo, como fase ecial.

De acordo com Hennen et al. (2005), esta ferrugem tem sido registrada sobre os seguintes gêneros de bambus: Bambusa, Dendrocalamus, Ochlandra, Oxytenanthera, Thyriostaschys e Gigantochloa. Este é o primeiro registro sobre o gênero Guadua.

11.2 Puccinia bambusarum Arthur, Bot. Gaz. 65:467. 1918.

Anamorfo: Uredoolyrae Henn., Hedwigia 43: 164. 1904. Material estudado: Sobre Pariana sp., BRASIL. AMAPÁ: Tartarugalzinho, Base da extrema, 14.XI.2005, H. Sotão \& L.C. Lobato H64 (III) (HAMAB, MG).

Esta espécie é reportada somente para Peru e Brasil (Amapá e Pará) (Hennen et al. 2005).

No inventário das espécies de Puccinia realizado por Sotão et al. (2001) em algumas áreas 

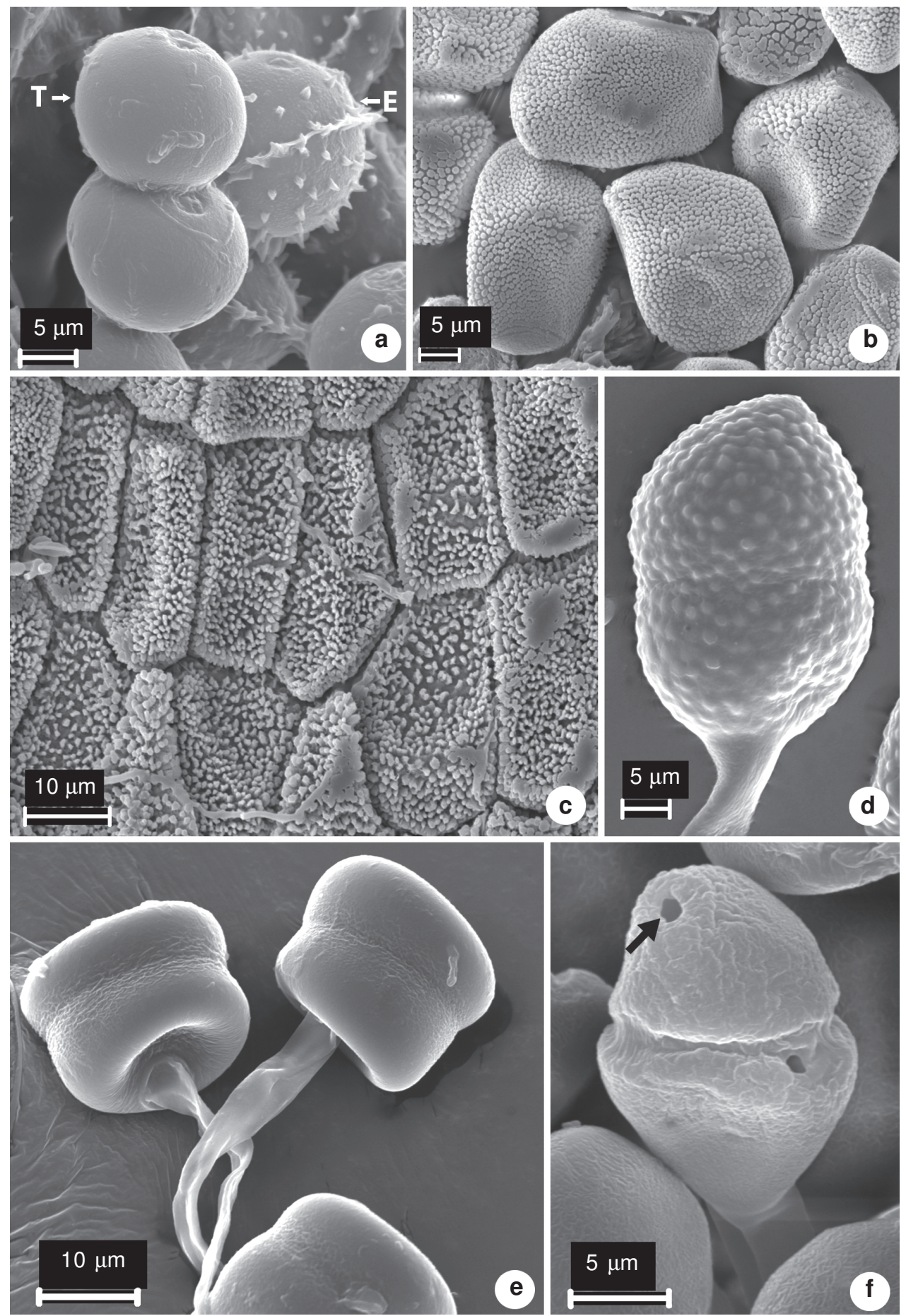

Figura 2 - a. Prospodium laevigatum J.F. Hennen \& Sotão, teliósporo (T) e eciósporo (E). b-d. Puccinia puta H.S. Jacks. \& Holw. ex F. Kern, Thurst. \& Whetzel - b. Urediniósporos de parede verrucosa; c. Parede interna verrucosa das células; d. Teliósporo de parede verrucosa. e. P. peperomiae J.C. Lindq. - teliósporos com inserção lateral dos pedicelos. f. P. lateritia Berk. \& M.A. Curtis - teliósporo destacando os poros germinativos (seta).

Figure 2 - a. Prospodium laevigatum JF Hennen \& Sotão, teliospore (T) and aeciospore (E). b-d. Puccinia puta H.S. Jacks. \& Holw. ex F. Kern, Thurst. \& Whetzel - b. Urediniospores wall verrucose; c. Inner spore wall verrucose; d. Teliospore wall verrucose. e. P. peperomiae J.C. Lindq. - teliospores with lateral attachment of pedicels. f. P. lateritia Berk. \& MA Curtis - teliospores with germ pore (arrow). 
do estado do Amapá, foram referidas as espécies $P$. bambusarum e P. obliquo-septata parasitando plantas da família Poaceae. Puccinia bambusarum difere-se de $P$. obliquo-septata por apresentar uredínios sem paráfises periféricas e parede apical dos teliósporos pouco espessada (2-3 mm), enquanto que $P$. obliquo-septata possui uredínios com paráfises periféricas e parede apical dos teliósporos bastante espessada (3-7 mm).

\section{Rubiaceae}

12.1 Puccinia lateritia Berk. \& M.A. Curtis, Jour. Acad. Nat. Sci. Philadelphia II, 2: 281. 1853. Fig. $2 f$ Material estudado: Sobre Borreria sp., BRASIL. AMAPÁ: Taratarugalzinho, Propriedade do Sr. Mimi, 29.VI.2006, I. França I01, I03 (III) (HAMAB, MG).

Esta espécie é amplamente distribuída, desde a Argentina até os Estados Unidos. No Brasil está registrada para os estados da Bahia, Minas Gerais, Pará, Rio de Janeiro e São Paulo (Hennen et al. 2005). Esta é a primeira citação desta espécie para o estado do Amapá.

Hennen et al. (2005) reportam quatro espécies de ferrugens sobre o gênero Borreria para o Brasil: Aecidium borreriicola H.S. Jacks. \& Holw., A. holwayi H.S. Jacks., Puccinia lateritia Berk. \& M.A. Curtis e Uredo borreriae (Henn.) F. Kern \& Whetzel. Puccinia lateritia é uma espécie microcíclica que produz apenas télios com teliósporos e mesosporos, enquanto $U$. borreriae e as duas espécies de Aecidium são anamorfos produzindo somente urediósporos e eciósporos, respectivamente.

\section{Smilacaceae}

13.1 Sphenospora smilacina Syd., Ann. Mycologici. 23: 318-319.1925.

Anamorfo: Uredo yurinaguasensis Henn., Hedwigia 43:164. 1904.

Material estudado: Sobre Smilax sp., BRASIL. AMAPÁ: Tartarugalzinho, Propriedade do Sr. Mimi, 29.VI.2006, I. França I10 (II) (HAMAB, MG).

Ocorre no Brasil (Amapá, Minas Gerais, Pará, Paraíba, Rio de Janeiro e São Paulo), Colômbia, Costa Rica, Equador, Guatemala, Honduras, México, Panamá, Peru, República Dominicana, Trinidad e Tobago e Venezuela (Jackson 1926; Gallegos \& Cummins 1981; Hennen et al. 2005).

Hennen et al. (2005) citam para o Brasil duas espécies de Uredinales sobreSmilax, Puccinia smilacis Schwein. e Sphenospora smilacina, que se diferenciam pelo tipo de septo dos teliósporos: horizontal em $P$. smilacis e vertical em $S$. smilacina.

\section{Agradecimentos}

Os autores agradecem ao MMA/IBAMA o financiamento, coordenação e apoio logístico do projeto Diagnósticos Abiótico, Biótico e Socioeconômico para subsidiar a elaboração do Plano de Manejo da Reserva Biológica do Lago Piratuba e a toda equipe do projeto; à Patrícia Pinha, Mírian Lucatelli, Iranildo Coutinho e toda a equipe do Instituto Chico Mendes/Amapá/ REBIO do Lago Piratuba a coordenação do projeto e organização das excursões; ao IEPA e MPEG as facilidades e infraestrutura oferecida; ao Dr. Joe F. Hennen a bibliografia cedida; aos colegas Luís Carlos Lobato, Carlos Alberto Silva e Priscila Sanjuan o apoio nas coletas e identificações Botânicas; a Jeferson Costa, a ajuda na composição das figuras; e à CAPES a concessão de bolsa de mestrado ao primeiro autor.

\section{Referências}

Albuquerque, F.C. 1971. Relação das espécies de Uredinales coletadas na Amazônia. Pesquisa Agropecuária Brasileira Série Agronômica 6: 147-150.

Barreto, R.W. \& Evans, H.C. 1995. The mycobiota of the weed Mikania micrantha in southern Brazil with particular reference to fungal pathogens for biological control. Mycological Research 99: 343-352.

Berndt, R.; Freire, F. \& Bastos, C.N. 2002. Crosssopsora piperis, a new rust species from Brazil. Mycotaxon 83: 265-268.

Buriticá, P. 1999a. La familia Phakopsoraceae en el Neotrópico III-Géneros: Batistopsora y Phakopsora. Revista de la Academia Colombiana de Ciencias Exactas, Físicas y Naturales 23: 271-305.

Buriticá, P. 1999b. La Familia Phakopsoraceae (Uredinales) en el Neotropico IV-Géneros: Crossopsora, Cerotelium, Phragmidiellay Catenulopsora. Revista de la Academia Colombiana de Ciencias Exactas, Físicas y Naturales 23: 407-431.

Carvalho Jr., A.A.; Hennen, J.F. \& Figueiredo, M.B. 2001. Primeira constatação do teleomorfo da ferrugem (Kweilingia divina) do bambu (Bambusa vulgaris) nas Américas. Summa Phytopathologica 27: 260-263.

Cummins, G.B. 1971. The rust fungi of cereals, grasses and bamboos. Springer-Verlag, New York. 570p.

Cummins, G.B. 1978. Rust fungi on legumes and composites in North America. University of Arizona Press, Tucson. 424p.

Cummins, G.B. \& Hiratsuka, Y. 2003. Illustrated genera of rust fungi. 3 ed. The American Phytopathological Society, St. Paul. 225p.

Dale, W.T. 1955. New species of Uredinales from Trinidad. Mycological Papers 59: 1-11. 
Evans, H.C. \& Ellison, C.A. 2005. The biology and taxonomy of rust fungi associated with the neotropical vine Mikania micrantha, a major invasive weed in Asia. Mycologia 97: 935-947.

Figueiredo, M.B. \& Hennen, J.F. 1998. Uredinales (ferrugens) no Brasil e no estado de São Paulo. Biológico 60: 17-29.

França, I.F. \& Sotão, H.M.P. 2009. Novos registros de ferrugens (Uredinales) sobre Fabaceae para o Brasil. Acta Botanica Brasilica 23: 860-863.

Gallegos, H.L. \& Cummins, G.B. 1981. Uredinales (Royas) de México. Vol. 1. Secretaria de Agricultura y Recursos Hidráulicos de México, Cualicán. 490p.

Hennen, J.F.; Figueiredo, M.B.; Carvalho Jr., A.A. \& Hennen, P.G. 2005. Catalogue of plant rust fungi (Uredinales) of Brazil. Disponível em <http:// www.jbrj.gov.br>. Acesso em 15 agosto 2005.

Hennen, J.F.; Hennen, M.M. \& Figueiredo, M.B. 1982. Índice das ferrugens (Uredinales) do Brasil. Arquivo do Instituto Biológico 49: 1-201.

Hennen, J.F. \& Mccain, J.W. 1993. New species and records of Uredinales from the Neotropics. Mycologia 85: 970-986.

Hennen, J.F. \& Sotão, H.M.P. 1996. New species of Uredinales on Bignoniaceae from Brazil. Sida 17: 173-184.

Hennen, J.F. \& Sotão, H.M.P. 1997. Aecidium maprouneae var. noncrassatum (Uredinales) sobre Maprouneae sp., novo táxon do estado do Amapá, Brasil. Fitopatologia Brasileira 22: 444-447.

Hennen, J.F.; Sotão, H.M.P. \& Hennen, M.M.W. 1998. The genus Diorchidium in the Neotropics. Mycologia 90: 1079-1086.
Hernández, J.R. 2006. First report of Puccinia puta on Ipomoea carnea subsp. fistulosa from Puerto Rico. Plant Disease 90: 245.

IBAMA. 2008. Unidades de Conservação. Reservas Biológicas. Unidade: Reserva Biológica doLago Piratuba. Disponível em <http://www.ibama.gov.br/ siucweb/ mostraUc.php?seqUc> Acesso em 03 novembro 2008.

Jackson, H.S. 1926. The rusts of South America based on the Holway colections - I. Mycologia 18: 139-162.

Jørstad, I. 1956. Uredinales from South America and Tropical North America. Arkiv For Botanik 2: 443-490.

Lindquist, J.C. 1982. Royas de la República Argentina y zonas limitrofes. Instituto Nacional de Tecnología Agropecuaria, Buenos Aires. 574p.

Sotão, H.M.P.; Hennen, J.F. \& Cavalcante, M.A. 2001. Uredinales do estado do Amapá: gênero Puccinia. Boletim do Museu Paraense Emílio Goeldi, série Botânica 17: 107-159.

Sotão, H.M.P.; França, I.F. \& Hennen, J.F. 2006. Fungos das Famílias Phakopsoraceae e Uropyxidaceae (Uredinales) da Floresta Nacional de Caxiuanã, Pará, Brasil. Hoehnea 33: 407-417.

Sotão, H.M.P.; Hennen, J.F.; França, I.F.; Freires, E.; Moura, M.F.; Martins Júnior, A.S.; Medeiros, P.S.; Silva, M.F.F. 2009. Ferrugens (Uredinales - Basidiomycota) da FLONA de Caxiuanã. In: Lisboa, P. (org.). Caxiuanã: desafios para conservação de uma floresta nacional na Amazônia. MPEG, Belém. Pp. 371-381.

Viégas, A.P. 1945. Alguns fungos do Brasil IV. Uredinales. Bragantia 5: 1-144.

Vieira, F.M.C.; Pereira, O.L. \& Barreto, R.W. 2004. First report of Coleosporium ipomoeae on Ipomoea fistulosa in Brazil. Fitopatologia Brasileira 29: 693. 